\title{
Evaluation of the Impact of Different Doses of Curcuma longa $L$. on Antioxidant Capacity: A Randomized, Double-Blind, Crossover Pilot Trial
}

\author{
Tatyanne Letícia N. Gomes $\mathbb{D}^{1}{ }^{1}$ Renata Santos S. Zenha, ${ }^{1}$ Alisson H. Antunes, ${ }^{2}$ \\ Flávia R. Faria, ${ }^{1}$ Kênnia R. Rezende $\mathbb{D}^{2},{ }^{2}$ Evandro L. de Souza $\mathbb{D}^{3},{ }^{3}$ and João F. Mota $(\mathbb{D})^{1}$ \\ ${ }^{1}$ School of Nutrition, Federal University of Goiás, Goiânia, Goiás, Brazil 74.605-080 \\ ${ }^{2}$ Laboratory of Biopharmacy and Pharmacokinetics, Federal University of Goiás, Goiânia, Goiás, Brazil 74.605-080 \\ ${ }^{3}$ Department of Nutrition, Health Sciences Center, Federal University of Paraíba, João Pessoa, Paraíba, Brazil 58.041-900 \\ Correspondence should be addressed to João F. Mota; jfemota@gmail.com
}

Received 5 August 2021; Accepted 25 November 2021; Published 14 December 2021

Academic Editor: Atef M. Al Attar

Copyright (C) 2021 Tatyanne Letícia N. Gomes et al. This is an open access article distributed under the Creative Commons Attribution License, which permits unrestricted use, distribution, and reproduction in any medium, provided the original work is properly cited.

Curcumin is a bioactive compound derived from Curcuma longa L. root, extensively studied due to its antioxidant and antiinflammatory properties. This study evaluates the effects of different doses of powdered C. longa root on antioxidant capacity in healthy men. In a pilot randomized, double-blinded, crossover experiment, we acutely administered a low dose (1.5 g, LCG), moderate dose (3.0 g, MCG), and high dose ( $6.0 \mathrm{~g}, \mathrm{HCG})$ of $C$. longa to nine healthy men. There were no differences in plasma curcumin levels $(p=0.593)$ and antioxidant capacity $(p=0.473)$ for time $\times$ group interactions. Plasma curcumin levels increased in all groups after 20 and $90 \mathrm{~min}$ of $C$. longa intake $(p<0.05)$. HCG had a lower postprandial incremental area under the antioxidant capacity curve than LCG or MCG $(p<0.01)$. A low dose of $C$. longa increased the antioxidant capacity in healthy men. However, plasma curcumin levels were not dose dependently affected.

\section{Introduction}

Curcuma longa $\mathrm{L}$. or turmeric is a perennial member of the Zingiberaceae family, widely used as a spice, food preservative, and dye $[1,2]$, and traditionally used as an anti-inflammatory, antioxidant, and antiseptic in Chinese and Indian medicine [3-5]. Curcumin, the major curcuminoid present in C. longa and responsible for the plant's yellow-orange coloration, is a low-molecular-weight polyphenol $[1,5,6]$. Curcumin pleiotropic activities have been attributed to its ability to influence multiple signaling pathways, including nuclear factor kappa B (NF- $\kappa \mathrm{B})$, protein kinases, growth factor pathways, NF-E2-related factor 2 (Nrf2), and the enzymes cyclooxygenase-2 and 5-lipoxygenase [4, 5]. Curcumin can reduce oxidative stress by inhibiting NF- $\kappa \mathrm{B}$-induced activation by phorbol-12-acetate-13-myristate (PMA) and hydrogen peroxide [1].
Previous experimental studies have reported the antioxidant effects of curcumin $[7,8]$. However, the results of clinical investigations on the antioxidant properties of curcumin are contradicting [9-11], which could be related to the administered doses. Excessive doses of antioxidant compounds can exert contrary action increasing oxidative stress and prooxidant activity $[12,13]$.

This study evaluates the acute effects of different doses of C. longa root powder on the antioxidant capacity of healthy men. The initial hypothesis was that individuals with the highest intake of turmeric would have a greater antioxidant capacity.

\section{Materials and Methods}

2.1. Study Design and Participants. This is a randomized, double-blind, crossover study. An Ethics Committee of the 
Federal University of Goiás (Goiânia, Brazil, n. 421.008) approved the research procedure. We conducted all experiments following institutional and governmental guidelines and regulations. All participants were informed about the risks and discomforts of this study before obtaining their written informed consent. This study was registered at http://ensaiosclinicos.gov.br (n. RBR-7tj48w).

We recruited participants through social media, adverts placed on college notice boards, and flyers. Participants were healthy males from 20 to 40 years old who could attend all study assessments. For characterization, body mass index was calculated as the ratio of weight to height squared $(\mathrm{kg} /$ $\mathrm{m}^{2}$ ) [14]. Exclusion criteria included the use of medications or nutritional supplements, alcohol ingestion during the study, the adoption of a diet or dietary restriction, and the use of turmeric as a spice at meals in the last seven days preceding the beginning of the study.

Twelve participants were assigned to either acutely ingest different amounts of powdered C. longa root in capsules or a 7-day washout period between treatments [15]. The high-dose group (HCG) ingested twelve capsules containing $500 \mathrm{mg}$ of $C$. longa $(6 \mathrm{~g})$, the moderate-dose group (MCG) ingested six capsules containing $500 \mathrm{mg}$ of C. longa $(3 \mathrm{~g})$ and six containing maltodextrin, and the low-dose group (LCG) ingested three capsules containing $500 \mathrm{mg}$ of C. longa (1.5 g) and nine capsules containing maltodextrin. Turmeric Producers Cooperative of Mara Rosa (Goiânia, Goiás, Brazil) supplied the C. longa root powder, and a pharmacy conducted encapsulation. The total amount of three major curcuminoids (curcumin, desmethoxycurcumin, and bisdemethoxycurcumin) found in the tested C. longa root powder was $4.3 \%$, as previously reported [16].

We randomized the intervention order using random number generating software. A researcher who was not involved in the outcome evaluations performed the randomization. The treatment conditions were unknown to all participants and the research team. Codes were revealed after the completion of all analyses.

We measured plasma curcumin after administering $C$. longa capsules at three different doses (LCG, MCG, and HCG), followed by nine venous blood collection time points: before (baseline) and 20, 30, 35, 40, 45, 60, 90, and $120 \mathrm{~min}$ after $C$. longa capsule administration. Furthermore, we measured the antioxidant activity percentage at $0,30,60,90$, and 120 min after C. longa capsule ingestion.

2.2. Quantification of Plasma Curcumin Levels. Blood samples were collected using a venous catheter in heparinized tubes at nine predetermined time points. Blood was immediately centrifuged $\left(1750 \mathrm{rpm}, 10 \mathrm{~min}, 4^{\circ} \mathrm{C}\right.$, centrifuge Beckman, Fullerton, CA, USA) for plasma separation. Subsequently, samples were frozen and stored at $-80^{\circ} \mathrm{C}$ in borosilicate test tubes coated in aluminum foil to avoid light exposure and degradation.

For every $2 \mathrm{ml}$ of plasma, $200 \mu \mathrm{l}$ of internal standard solution ( $\beta$-estradiol) and $1800 \mu \mathrm{l}$ of chloroform were added, vortexed $(10 \mathrm{~s})$, shacked $(5 \mathrm{~min})$, and centrifuged $(5500 \mathrm{rpm}$, $\left.10 \mathrm{~min}, 4^{\circ} \mathrm{C}\right)$. After phase separation, an aliquot $(1000 \mu \mathrm{l})$ of the organic phase was extracted and transferred to another clean borosilicate tube, followed by solvent evaporation under a nitrogen atmosphere for dryness $(10 \mathrm{~min})$. The dry residue was rehydrated in acetonitrile $(500 \mu \mathrm{l})$, transferred to vials, and injected into a high-performance liquid chromatography (HPLC) system. Duplicates of daily calibration curves were created for curcumin quantification and quality control samples at three concentration levels (high, medium, and low) for analytical assurance. We determined the unknown plasma curcumin concentrations using HPLC according to a validated bioanalytical method, following the European Medicines Agency guidelines [17]. All extraction procedures were conducted under low light incidence to prevent curcumin degradation.

2.3. Measurement of Antioxidant Capacity. The antioxidant capacity was measured using a previously described method [18], making minor modifications. The method is based on electron transfer where 2,2-diphenyl-1-picryl-hydrazine (DPPH, purple) is reduced by the action of an antioxidant to form diphenyl-picryl-hydrazine (yellow) with a consequent reduction in absorption, which can be measured by decreasing absorbance.

Initially, we prepared an $80 \%$ methanol solution to dilute $0.002 \mathrm{~g}$ DPPH in a $25 \mathrm{ml}(200 \mu \mathrm{mol} / \mathrm{l})$ volumetric flask. After dilution, the DPPH solution was sonicated for $20 \mathrm{~min}$ to achieve complete solubilization. Then, $80 \mu \mathrm{l}$ of methanol representing a control sample and $80 \mu \mathrm{l}$ of methanol deproteinized plasma were dispensed into a microplate. The sample and control blank samples were repeated on the next row of the microplate. Then, $160 \mu \mathrm{l}$ of DPPH solution was dispensed in the first row, and $160 \mu \mathrm{l}$ of $80 \%$ methanol was dispensed in the second row, consecutively intercalated. After incubating samples for $50 \mathrm{~min}$, we measured the absorbance at $517 \mathrm{~nm}$. We calculated the percentage DPPH reduction using the following formula: percentage $\mathrm{DPPH}$ reduction $=$ $[1$ - (the sample - A white/the control - A white $)] \times 100$.

2.4. Statistical Analysis. We used Statistical Package for the Social Sciences (SPSS) statistical software v.25 (SPSS Inc., Chicago, IL, USA) for the statistical analyses. GraphPad Prism 5.0 (GraphPad Software, Inc., San Diego, CA) was used to create the graphs. Results are presented as mean \pm standard deviation. We used the Kolmogorov-Smirnov test to determine the normality of the data normality. We used a two-way analysis of variance test (groups $\times$ times) with repeated measurements to analyze plasma curcumin and antioxidant capacity. When a significant interaction was detected, Bonferroni's multiple comparison method was used. We used the trapezoidal method to compare postprandial responses between conditions with the incremental area under the curve (iAUC) [19]. Effect sizes (ES) were calculated using Cohen's formula, which were then classified as small $(d=0.2)$, medium $(d=0.5)$, or large $(d=0.8)$. A $p$ value of $<0.05$ was considered statistically significant.

\section{Results}

Twelve out of the 15 interested patients were eligible and initially enrolled in this study. Three participants withdrew for 


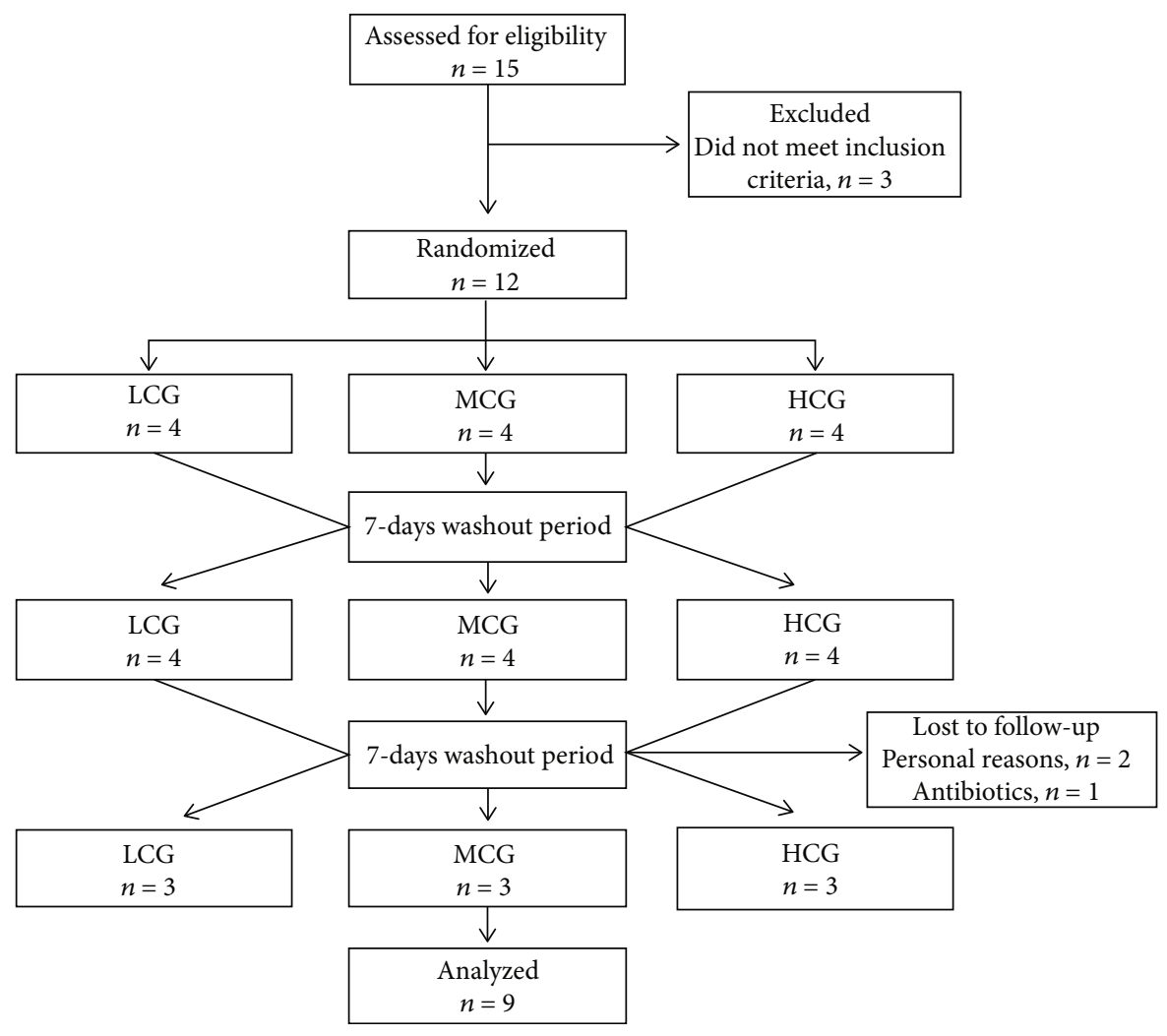

Figure 1: Participant flow diagram.

personal reasons $(n=2)$ and antibiotic use $(n=1)$ (Figure 1$)$. The average age and body mass index of participants were $27 \pm 1 \mathrm{y}$ and $23.31 \pm 0.75 \mathrm{~kg} / \mathrm{m}^{2}$, respectively.

There were no differences in plasma curcumin levels $[F(1.66,8.32)=0.88, p=0.637]$ and \%DPPH $[F(2.76$, $22.09)=1.46, p=0.473]$ for time $\times$ group interaction. Plasma curcumin levels varied within the group, showing a significant increase after 20 and $90 \mathrm{~min}$ (all groups $p<0.05$, Figure 2(a)). Ingestion of a high dose of $C$. longa did not increase \%DPPH values (Figure 2(b)). However, LCG and MCG groups had better iAUC for \%DPPH compared to the HCG group $[F(2,24)=107.24, p<0.01, \mathrm{ES}=0.90$ and 0.82 , respectively]. The maximum plasma curcumin level was $41.63 \pm 87.93 \mathrm{ng} / \mathrm{ml}$, found in the LCG group. The maximum plasma curcumin-level time was similar for the LCG and MCG groups (at $90 \mathrm{~min}$ ), while it was found at $40 \mathrm{~min}$ in the HCG group (Table 1).

\section{Discussion}

In this well-controlled pilot study, we discovered that the ingestion of higher doses of $C$. longa had no significant influence on antioxidant capacity and curcumin bioavailability in healthy men. Furthermore, a low intake of $C$. longa $(1.5 \mathrm{~g})$ may be more effective in inducing higher antioxidant capacity. These results support the notion that the cause of oxidative stress is an imbalance between the body's prooxidant and antioxidant compounds. Thus, even nutrients with antioxidant potential could exert contrary action in the body when administered in excess [13]. Furthermore, curcumin is a nutritional hormone that follows hormonal pathways because the response to hormonal dose is biphasic and characterized by a dose-response relationship divided into two dose zones: low-dose area with stimulatory responses and high-dose area with inhibitory and adverse reactions [20].

In this study, plasma curcumin levels were undetectable at some collection points, exhibiting fluctuations throughout the study. These fluctuations could be attributed to curcumin metabolism, as the absorption of this compound is followed by conjugation in the liver (glucuronidation and sulfation) and excretion through the gallbladder. Curcumin deconjugation occurs in the intestine due to the action of intestinal bacteria and is reabsorbed [21]. Curcumin is also rapidly absorbed, metabolized, and distributed to tissues within the first few minutes [22]. Therefore, low plasma levels observed in studies using oral curcumin may be not only due to its malabsorption and rapid metabolism but also due to rapid tissue distribution [23].

Garcea et al. [24] discovered trace amounts of curcumin $(<1 \mathrm{ng} / \mathrm{ml})$ in patients with colorectal cancer after ingestion of $3.6 \mathrm{~g}$ of curcumin, which is consistent with the results of this study. Similarly, another study reported that turmeric extract supplementation (440-2200 mg/d; 36-180 mg of curcumin) in 15 patients with advanced colorectal cancer for 29 days did not increase curcumin levels or the metabolites in the blood or urine [25]. However, consumption of $>8 \mathrm{~g}$ of curcumin per day did not increase plasma curcumin levels in proportion to the administered doses in healthy volunteers $[26,27]$. Thus, plasma curcumin levels did not increase 


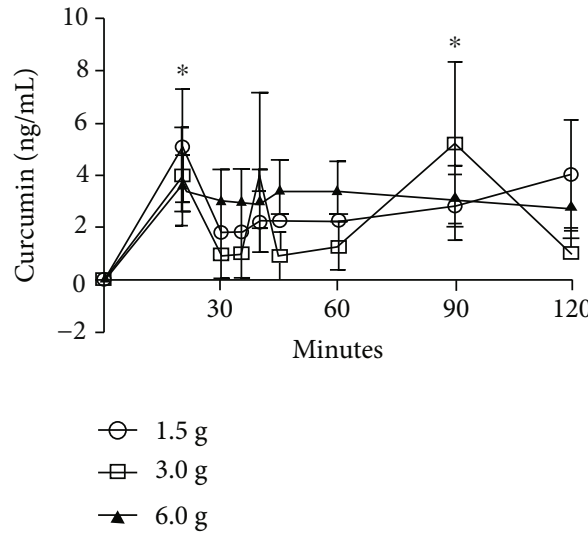

(a)

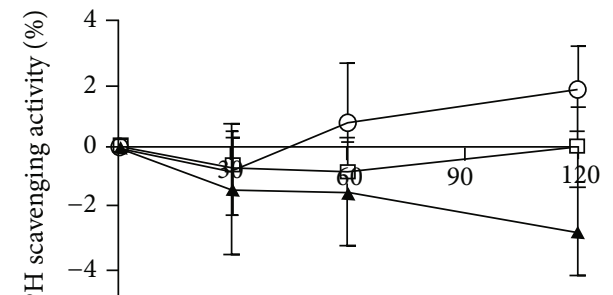

Minutes

$$
\begin{aligned}
& -1.5 \mathrm{~g} \\
& \square 3.0 \mathrm{~g} \\
& -6.0 \mathrm{~g}
\end{aligned}
$$

(b)

FIgURE 2: Plasma curcumin levels (a) and antioxidant capacity (b) in healthy men after acute ingestion of different doses of Curcuma longa L. There were no group $\times$ time interactions. ${ }^{*} p<0.05$ vs. baseline in all groups.

TABLE 1: Pharmacokinetic parameters for plasma curcumin levels

\begin{tabular}{|c|c|c|c|}
\hline & $\begin{array}{c}\text { LCG } \\
(1.5 \mathrm{~g})\end{array}$ & $\begin{array}{l}\text { MCG } \\
(3.0 \mathrm{~g})\end{array}$ & $\begin{array}{l}\text { HCG } \\
(6.0 \mathrm{~g})\end{array}$ \\
\hline$C_{\max }(\mathrm{ng} / \mathrm{ml})$ & $41.63 \pm 87.93$ & $41.20 \pm 12.35$ & $2.84 \pm 8.53$ \\
\hline$T_{\max }$ & $90 \mathrm{~min}$ & $90 \mathrm{~min}$ & $40 \mathrm{~min}$ \\
\hline
\end{tabular}
in healthy men induced by acute ingestion of different doses of Curcuma longa $\mathrm{L}$.

proportionally to the administered dose, probably due to absorption system saturation.

In this study, the maximum plasma curcumin-level time was $90 \mathrm{~min}$, which was longer than that (30-45 $\mathrm{min})$ found by Pawar et al. [22]. However, these authors used curcumin in conjunction with lipid formulation. Curcumin is a highly lipophilic compound that could be absorbed faster when administered with liposomes, micelles, or phospholipid complexes $[28,29]$. Curcumin absorption may reduce even further when fasting because bile synthesis required for solubilization of hydrophobic compounds is reduced [28]. In our study, capsules were ingested while fasting, which may have reduced curcuminoid absorption.

C. longa has been reported as a potent antioxidant and anti-inflammatory herb [30]. However, there are still disagreements among studies regarding these effects, which may be related to curcumin dose in tested supplements. It has been demonstrated that a high concentration of antioxidants can induce prooxidant activity [13]. Supporting this finding, an early experimental study showed that curcumin concentration dependently exhibits anti- and prooxidant effects [12]. In a previous study [31], a low dose of a curcumin-lipid preparation $(80 \mathrm{mg} /$ day $)$ induced various potentially health-promoting effects in healthy middle-aged individuals, including free radical scavenging capacity, increased plasma catalase activity and plasma myeloperoxidase levels, and lower levels of C-reactive protein and plasma nitric oxide [31]. Curcumin administration as a supplement has been considered safe, with clinical trials in humans revealing no dose-limiting toxicity when administered up to $12 \mathrm{~g} /$ day [26].

This study had some limitations, such as a small number of participants despite being a pilot study. Furthermore, we evaluated the effects on healthy men without metabolic stress, being possible that individuals with oxidative stress could need higher doses of an antioxidant to reach positive effects on antioxidant capacity.

\section{Conclusions}

In conclusion, a low dose of $C$. longa enhanced the antioxidant capacity in healthy men; however, plasma curcumin levels were not dose-dependently affected. High doses of $C$. longa might negatively impact the antioxidant capacity in healthy men. Further studies with a larger sample size and longer duration are required to corroborate these results.

\section{Data Availability}

Data is available on request.

\section{Conflicts of Interest}

There is none to declare.

\section{Authors' Contributions}

TLNG, KRR, FRF, and JFM contributed to the study design. TLNG, RSS, FRF, and AHA collected the data of the manuscript. TLNG, RSS, ELS, and JFM performed the literature search and participated in the discussion of the manuscript. TLNG, RSS, and AHA drafted the manuscript. ELS, KRR, and JFM participated in a critical discussion of data and reviewed the manuscript. All authors read and approved the final version of the article. 


\section{Acknowledgments}

We would like to thank all the volunteers and Dr. Patricia Botelho for the DPPH analysis. The study was supported by the National Council for Scientific and Technological Development (CNPq), Brazil (484023/2013-6). TLNG would like to thank $\mathrm{CNPq}$ for the scholarship. J.F.M. and E.LS. have received support from CNPq (no. 305082/2019-1 and 301889/2019-8, respectively).

\section{References}

[1] J. S. Jurenka, "Anti-inflammatory properties of curcumin, a major constituent of Curcuma longa: a review of preclinical and clinical research," Alternative Medicine Review, vol. 14, pp. 141-153, 2009.

[2] R. A. Sharma, A. J. Gescher, and W. P. Steward, "Curcumin: the story so far," European Journal of Cancer, vol. 41, no. 13, pp. 1955-1968, 2005.

[3] P. Basnet and N. Skalko-Basnet, "Curcumin: an antiinflammatory molecule from a curry spice on the path to cancer treatment," Molecules, vol. 16, no. 6, pp. 4567-4598, 2011.

[4] H. Hatcher, R. Planalp, J. Cho, F. M. Torti, and S. V. Torti, "Curcumin: from ancient medicine to current clinical trials," Cellular and Molecular Life Sciences, vol. 65, no. 11, pp. 1631-1652, 2008.

[5] H. Zhou, C. S. Beevers, and S. Huang, "The targets of curcumin," Current Drug Targets, vol. 12, no. 3, pp. 332-347, 2011.

[6] K.-Y. Yang, L.-C. Lin, T.-Y. Tseng, S.-C. Wang, and T.-H. Tsai, "Oral bioavailability of curcumin in rat and the herbal analysis from Curcuma longa by LC-MS/MS," Journal of Chromatography $B$, vol. 853, no. 1-2, pp. 183-189, 2007.

[7] Z. M. M. Al-Rubaei, T. U. Mohammad, and L. K. Ali, "Effects of local curcumin on oxidative stress and total antioxidant capacity in vivo study," Pakistan Journal of Biological Sciences, vol. 17, no. 12, pp. 1237-1241, 2014.

[8] A. Rajasekar and T. Devasena, "Facile synthesis of curcumin nanocrystals and validation of its antioxidant activity against circulatory toxicity in Wistar rats," Journal of Nanoscience and Nanotechnology, vol. 15, no. 6, pp. 4119-4125, 2015.

[9] C. Blanton and B. Gordon, "Effect of morning vs. evening turmeric consumption on urine oxidative stress biomarkers in obese, middle-aged adults: a feasibility study," International Journal of Environmental Research and Public Health, vol. 17, no. 11, p. 4088, 2020.

[10] F. Drobnic, J. Riera, G. Appendino et al., "Reduction of delayed onset muscle soreness by a novel curcumin delivery system (Meriva $\left.{ }^{\circledR}\right)$ : a randomised, placebo-controlled trial," Journal of the International Society of Sports Nutrition, vol. 11, no. 1, 2014.

[11] R. Tabrizi, S. Vakili, M. Akbari et al., "The effects of curcumincontaining supplements on biomarkers of inflammation and oxidative stress: a systematic review and meta-analysis of randomized controlled trials," Phytotherapy Research, vol. 33, no. 2, pp. 253-262, 2019.

[12] C. Li, X. Miao, F. Li et al., "Curcuminoids: implication for inflammation and oxidative stress in cardiovascular diseases," Phytotherapy Research, vol. 33, no. 5, pp. 1302-1317, 2019.

[13] A. Rahal, A. Kumar, V. Singh et al., "Oxidative stress, prooxidants, and antioxidants: the interplay," BioMed Research International, vol. 2014, Article ID 761264, 19 pages, 2014.
[14] CONSULTATION WHO, Definition, diagnosis and classification of diabetes mellitus and its complications: report of a WHO consultation. Part 1, diagnosis and classification of diabetes mellitus, World Health Organization, 1999.

[15] R. Jäger, R. P. Lowery, A. V. Calvanese, J. M. Joy, M. Purpura, and J. M. Wilson, "Comparative absorption of curcumin formulations," Nutrition Journal, vol. 13, no. 1, 2014.

[16] A. H. Antunes, F. R. Faria, J. F. Mota, M. F. Santiago, A. C. Kogawa, and K. R. Rezende, "Bioanalytical method by HPLCFLD for curcumin analysis in supplemented athletes," Saudi Pharmaceutical Journal, vol. 28, no. 5, pp. 599-606, 2020.

[17] European Medicines Agency, “Annual report 2011: overview of the Agency's contribution to science, medicines and health in the European Union," European Medicines Agency, 2011.

[18] G. Bobo-García, G. Davidov-Pardo, C. Arroqui, P. Vírseda, M. R. Marín-Arroyo, and M. Navarro, "Intra-laboratory validation of microplate methods for total phenolic content and antioxidant activity on polyphenolic extracts, and comparison with conventional spectrophotometric methods," Journal of the Science of Food and Agriculture, vol. 95, no. 1, pp. 204209, 2015.

[19] J. C. Pruessner, C. Kirschbaum, G. Meinlschmid, and D. H. Hellhammer, "Two formulas for computation of the area under the curve represent measures of total hormone concentration versus time-dependent change," Psychoneuroendocrinology, vol. 28, no. 7, pp. 916-931, 2003.

[20] E. Agathokleous and E. J. Calabrese, "Hormesis: the dose response for the 21st century: the future has arrived," Toxicology, vol. 425, article 152249, 2019.

[21] M. Metzler, E. Pfeiffer, S. I. Schulz, and J. S. Dempe, "Curcumin uptake and metabolism," BioFactors, vol. 39, no. 1, pp. 14-20, 2013.

[22] Y. B. Pawar, B. Munjal, S. Arora et al., "Bioavailability of a lipidic formulation of curcumin in healthy human volunteers," Pharmaceutics, vol. 4, no. 4, pp. 517-530, 2012.

[23] L. Shen, C.-C. Liu, C.-Y. An, and H.-F. Ji, "How does curcumin work with poor bioavailability? Clues from experimental and theoretical studies," Scientific Reports, vol. 6, no. 1, article 20872, 2016.

[24] G. Garcea, D. P. Berry, D. J. Jones et al., "Consumption of the putative chemopreventive agent curcumin by cancer patients: assessment of curcumin levels in the colorectum and their pharmacodynamic consequences," Cancer Epidemiology, Biomarkers \& Prevention, vol. 14, pp. 120-125, 2005.

[25] R. A. Sharma, H. R. McLelland, K. A. Hill et al., "Pharmacodynamic and pharmacokinetic study of oral curcuma extract in patients with colorectal cancer," Clinical Cancer Research, vol. 7, pp. 1894-1900, 2001.

[26] C. D. Lao, M. T. Ruffin, D. Normolle et al., "Dose escalation of a curcuminoid formulation," BMC Complementary and Alternative Medicine, vol. 6, no. 1, 2006.

[27] S. K. Vareed, M. Kakarala, M. T. Ruffin et al., "Pharmacokinetics of curcumin conjugate metabolites in healthy human subjects," Cancer Epidemiology, Biomarkers \& Prevention, vol. 17, no. 6, pp. 1411-1417, 2008.

[28] P. Anand, A. B. Kunnumakkara, R. A. Newman, and B. B. Aggarwal, "Bioavailability of curcumin: problems and promises," Molecular Pharmaceutics, vol. 4, no. 6, pp. 807-818, 2007.

[29] S. Singh, S. Jamwal, and P. Kumar, "Piperine enhances the protective effect of curcumin against 3-np induced neurotoxicity: 
possible neurotransmitters modulation mechanism," Neurochemical Research, vol. 40, no. 8, pp. 1758-1766, 2015.

[30] C. Araújo and L. Leon, "Biological activities of Curcuma longa L," Memórias do Instituto Oswaldo Cruz, vol. 96, no. 5, pp. 723-728, 2001.

[31] R. A. DiSilvestro, E. Joseph, S. Zhao, and J. Bomser, "Diverse effects of a low dose supplement of lipidated curcumin in healthy middle aged people," Nutrition Journal, vol. 11, no. $1,2012$. 